\title{
The Association between Hypertension and Dementia in the Elderly
}

\author{
Michiya Igase, ${ }^{1}$ Katsuhiko Kohara, ${ }^{1}$ and Tetsuro Miki ${ }^{1,2}$ \\ ${ }^{1}$ Department of Geriatric Medicine, Ehime University Graduate School of Medicine, Toon City, Ehime 791-0295, Japan \\ ${ }^{2}$ Proteo-Medicine Research Center, Ehime University, Ehime 791-0295, Japan \\ Correspondence should be addressed to Michiya Igase, migase@m.ehime-u.ac.jp
}

Received 12 August 2011; Accepted 19 September 2011

Academic Editor: Blas Gil Extremera

Copyright ( $) 2012$ Michiya Igase et al. This is an open access article distributed under the Creative Commons Attribution License, which permits unrestricted use, distribution, and reproduction in any medium, provided the original work is properly cited.

\begin{abstract}
Hypertension (HT) and dementia are common disorders in the elderly. HT in the elderly is associated with increased occurrence rates of dementia including Alzheimer's disease $(\mathrm{AD})$ and vascular dementia $(\mathrm{VaD})$. In connection to this, some studies have suggested that HT in old age correlates with the pathogenesis of dementia. Since HT is potentially reversible, a number of randomized trials have examined whether antihypertensive treatment may help in preventing dementia occurrence. We review five studies, all using subjects 60 years or older, which investigated different antihypertensive pharmacological treatments. Data from two trials (Syst-Eur, PROGRESS) open the way toward the prevention of dementia (AD or VaD) by antihypertensive treatments. In the Syst-Eur study, with the dihydropyridine calcium antagonists, a reduction in both types of dementia was demonstrated (risk reduction 55\%). The PROGRESS study showed that the use of angiotensin-converting enzyme inhibitors (ACEIs), with or without diuretics, resulted in decrease incidence of stroke-related dementia (risk reduction 19\%), but dementia without stroke was not reduced. In contrast, the SHEP trial, treatment with a chlorthalidone-based antihypertensive regimen, did not significantly reduced the incidence of dementia. The SCOPE study (candesartan or hydrochlorothiazide versus placebo) and the HYVET-COG study (indapamide or perindopril versus placebo) found no significant difference between the active treatment and placebo group on the incidence of dementia. We found conflicting results regarding treatment benefits in dementia prevention. Recent clinical trials and studies on animal models suggest that blockades of RAS system could have reduced cognitive decline seen in Alzheimer's disease and vascular dementia. Future trials primarily designed to investigate the effects of antihypertensive agents on impaired cognition are needed.
\end{abstract}

\section{Introduction}

In general, the risk of HT, which is defined as a systolic blood pressure (SBP) $\geq 140 \mathrm{~mm} \mathrm{Hg}$ and/or a diastolic blood pressure (DBP) $\geq 90 \mathrm{~mm} \mathrm{Hg}$ [1], increases with advancing age. In fact, the prevalence of HT in individuals 60 years and older is double that of those aged $49-59$ years. In Framingham study, $90 \%$ of all 65 -year-old men and women with normal BP later developed HT [2]. This condition carries a very high risk for cerebrovascular disease (CVD) as well as coronary heart disease (CHD) [3]. Dementia is one of the most important neurological disorders in the elderly. Many studies have identified HT as marker for the pathogenesis of dementia $\mathrm{AD}$ and $\mathrm{VaD}$, while longitudinal studies have suggested that HT is associated with a higher incidence of dementia in old age. It has been observed that long-standing HT may lead to severe atherosclerosis and impaired cerebrovascular autoregulation, which in turn is thought to correlate with dementia [4]. For these reasons, several studies have investigated whether antihypertensive treatment may retard cognitive decline or dementia [5-9]. Although the importance of lowering BP in HT subjects is well known, the relationship between HT and cognitive function is controversial.

\section{HT in the Elderly and the Risk of Dementia}

To this date, the associations between $\mathrm{BP}$ and dementia have been inconclusive. Considering that the incidence of dementia among the elderly population is rising rapidly worldwide [10] and accumulating evidence that HT may contribute to the development of both $\mathrm{AD}$ and $\mathrm{VaD}$ [11], there is a reason to believe effective management of HT may 
translate into major health benefits through the protection of dementia. HT has long been known to cause CV [12]. Midlife HT ranks as an important modifiable risk factor for late-life cognitive decline [13], mild cognitive impairment (MCI) $[14,15]$, and $\mathrm{VaD}[16,17]$. In longitudinal cohort studies, elevated BP is associated with cognitive decline although some cross-sectional studies showed mixed relationships between higher BP and cognition, with many studies showing no correlation or even J- or U-shaped associations [18]. Findings from these prospective cohort studies for DBP and cognitive decline are less consistent; however, many have reported a similar inverse relation. The data on the role of BP and HT in later life are not consistent, leaving open the issue of BP treatment in elderly people. The controversy about the association between HT in the elderly and dementia arises because the longitudinal relationship between $\mathrm{BP}$ and cognitive change is sensitive to the effects of age, duration of followup and hypertensive treatment status, comorbidity with CVD and CHD, and possibly subclinical dementia [19]. More recently, a total of 668 communitydwelling Japanese individuals without dementia, aged 65 to 79 years, were followed up for 17 years, and examined the associations of late-life and midlife $\mathrm{HT}$ with the risk of $\mathrm{AD}$ and $\mathrm{VaD}[20]$. During the followup, 123 developed $\mathrm{AD}$, and 76 subjects experienced $\mathrm{VaD}$, and the age- and sex-adjusted incidence of $\mathrm{VaD}$ significantly increased with elevated midlife BP levels regardless of late-life BP levels. There were not a significant association between BP levels and AD. Li et al. [21] followed a total of 837 subjects with MCI for 5 years, 298 subjects converted to $\mathrm{AD}$, while 352 remained MCI at the end of the followup. Subjects with HT increased the risk of dementia conversion. Given their results, treatment of HT was associated with a reduced progression in $\mathrm{MCI}$ to $\mathrm{AD}$ dementia.

\section{Can Control of HT Protect against Dementia?}

Despite the speculated relationship between HT and dementia, clinical trials examining the preventive effects of antihypertensive therapy on dementia have been inconclusive (Table 1). Among five randomized double-blind placebocontrolled trials surveying antihypertensive treatments and dementia, four (Syst-Eur, PROGRESS, SCOPE, and HYVETCOG) used the Mini-Mental State Examination (MMSE), a widely used screening instrument for cognitive impairment, to assess cognitive function. In the SHEP, cognitive screening was performed by a short-comprehensive assessment and referral evaluation (short CARE) questionnaire. These five studies are described below.

3.1. Syst-Eur. The systolic hypertension in Europe study (Syst-Eur) investigated whether antihypertensive treatment in elderly patients with isolated systolic hypertension (ISH) led to a significant change in stroke morbidity and mortality. Syst-Eur investigated the effects of a calcium channel blocker (CCB; $10-40 \mathrm{mg} /$ day nitrendipine). If necessary, nitrendipine was combined with an ACEI $(5-20 \mathrm{mg} / \mathrm{d}$ enalapril maleate) and/or a diuretic (12.5-25 mg/day hydrochlorothiazide). Participants had no dementia and were at least
60 years old. Their SBP at the beginning of the trial was between 160 and $219 \mathrm{~mm} \mathrm{Hg}$, and their DBP was below $95 \mathrm{~mm} \mathrm{Hg}$. Antihypertensive therapy began immediately after randomization in the active treatment group, but only after termination of the double-blind trial in the control patients. The mean difference in BP between treatment groups and the control was $7.0 \mathrm{~mm} \mathrm{Hg} \mathrm{SBP}$ and $3.2 \mathrm{~mm} \mathrm{Hg}$ DBP; the rates of dementia for patients in the active treatment groups and the control groups were 3.3 and 7.4 cases per 1.000 patient-years (relative risk reduction: 55\%; 95\% CI: 24-73\%), respectively, which is significant.

In Syst-Eur, because active treatment using a $\mathrm{CCB}$ resulted in a $42 \%$ decrease in the primary end point of fatal and nonfatal stroke, only 2418 of the 4695 randomly assigned patients participated in a substudy on dementia. Compared with the control group, the portion of the treatment group that received only CCB $(60 \%)$ had a significantly reduced risk of dementia (55\%). Interestingly, while the total incidence of dementia was 64 cases, 41 of these showed AD. Therefore, Syst-Eur suggests using a CCB to lower BP may protect against dementia, particularly $\mathrm{AD}$, in elderlywith ISH.

3.2. PROGRESS. The perindopril protection against recurrent stroke study (PROGRESS) was a trial involving 6105 patients, all with prior stroke or transient ischemic attack. Participants were assigned to either active treatment (perindopril for all participants plus indapamide for those with neither an indication nor a contraindication to a diuretic) or a matching placebo. The mean difference in BP between the two groups was $9.0 \mathrm{~mm} \mathrm{Hg} \mathrm{SBP}$ and $4.0 \mathrm{~mm} \mathrm{Hg}$ DBP; the rates of dementia patients in the active treatment groups and the control groups were $6.3 \%$ and $7.1 \%$ (relative risk reduction: $12 \%$; $95 \%$ CI: -8 to $28 \%$ ), respectively, which is insignificant. However, the rates of cognitive decline were 9.1 and $11.0 \%$ (risk reduction: 19\%; 95\% CI: 4-32\%), respectively, which is significant.

3.3. SHEP. The systolic hypertension in the elderly program (SHEP) study was a trial conducted over an average 5year follow-up and involved 16 academic clinics. Among the 447.921 candidates aged 60 years and older screened, 4736 $(1.06 \%)$ were chosen for the study. SBP at baseline ranged from 160 to $219 \mathrm{~mm} \mathrm{Hg}$, while DBP was less than $90 \mathrm{~mm} \mathrm{Hg}$. Participants were randomized into either an active antihypertensive drug therapy or a matching placebo group. Active treatment consisted of a diuretic $(12.5-25 \mathrm{mg} /$ day chlorthalidone) for step 1 and a beta blockade (25-50 mg/day atenolol) for step 2. If atenolol was contraindicated, 0.05 to $0.10 \mathrm{mg}$ reserpine was used instead. The cohort mean difference in BP between the treatment groups and placebo was $12.0 \mathrm{~mm} \mathrm{Hg}$ SBP and $4.0 \mathrm{~mm} \mathrm{Hg} \mathrm{DBP}$; the rates of dementia for the active treatment group and the control group were 3.6 and 4.2 cases per 1.000 patient-years (relative risk reduction: $14 \%$; $95 \%$ CI: -26 to $54 \%$ ), respectively, which is insignificant.

3.4. SCOPE. The study on cognition and prognosis in the elderly (SCOPE) was a prospective study conducted from 
TABLE 1: Randomized controlled trials about antihypertensive treatments and dementia/cognitive decline.

\begin{tabular}{lll}
\hline Study setting & Participants and follow up & Treatment \\
\hline & & \\
& & CCB (nitrendipine) with \\
Systolic hypertension in Europe & $\begin{array}{l}2.418 \text { systolic hypertensives; } \\
\text { mean age 70 years, followup }\end{array}$ & $\begin{array}{l}\text { possible addition of ACE-I } \\
\text { (enalapril), diuretic }\end{array}$ \\
study (Syst- Eur) [5] & 3.9 years & (hydrochlorothiazide), or \\
& &
\end{tabular}

Test Main results

Mean difference in BP between

treatment groups and the control was $7.0 \mathrm{~mm} \mathrm{Hg} \mathrm{SBP}$ and $3.2 \mathrm{~mm} \mathrm{Hg}$ DBP. Rates of Systolic hypertension in Europe mean age 70 years, followup 3.9 years possible addition of ACE-I

(hydrochlorothiazide), or

both versus placebo

MMSE dementia for patients in the active treatment groups and the control groups were 3.3 and 7.4 cases per 1.000 patient-years (relative risk reduction: 55\%), respectively. Significant.

Mean difference in BP between treatment groups and the control was $9.0 \mathrm{~mm} \mathrm{Hg} \mathrm{SBP}$ and $4.0 \mathrm{~mm} \mathrm{Hg}$ DBP. Rates of

The perindopril protection against recurrent stroke study (PROGRESS) [6]
6.105 subjects with prior stroke or transient ischemic attack; mean age 64 years, followup 3.9 years
ACE-I (perindopril) with possible addition of diuretic (indapamide) versus placebo
MMSE cognitive decline for patients in the active treatment groups and the control groups were 11.0 and 9.1\% (relative risk reduction: $19 \%)$, respectively. Significant.

Mean difference in BP between treatment groups and the control was $12.0 \mathrm{~mm} \mathrm{Hg} \mathrm{SBP}$ and $4.0 \mathrm{~mm} \mathrm{Hg}$ DBP. Rates of Diuretic (chlorthalidone) with possible addition of $\beta$ blocker (atenolol) or sympathetic nervous blocker (reserpine) versus placebo

Short dementia incidence for patients

CARE in the active treatment groups and the control groups were 3.6 and 4.2 cases per 1.000
4.736 systolic hypertensives; mean age 72 years, followup 4.5 years
Systolic hypertension in the
elderly program (SHEP) [7]
4.964 hypertensives; SBP160-170/DBP

Study on cognition and prognosis in the elderly (SCOPE) [8]
90-99 mm Hg; aged 70- 89, followup 3.97 years
ARB (candesartan) versus placebo; open-label antihypertensive drugs were added to both groups patient-years (relative risk reduction: $14 \%$ ), respectively. Not significant.

Mean difference in BP between treatment groups and the control was $3.2 \mathrm{~mm} \mathrm{Hg} \mathrm{SBP}$ and $1.6 \mathrm{~mm} \mathrm{Hg} \mathrm{DBP}$. Rates of dementia incidence for patients in the active treatment groups and the control groups were 6.3 and 6.8 cases per 1.000 patient-years, respectively. Not Significant.

Mean difference in BP between treatment groups and the control was $15 \mathrm{~mm} \mathrm{Hg} \mathrm{SBP}$ and $5.9 \mathrm{~mm} \mathrm{Hg}$ DBP. Rates of dementia incidence for patients in the active treatment groups and the control groups were 33 and 38 cases per 1.000 patient-years (hazard ratio 0.86 ). respectively. Not significant.

BP: blood pressure, SBP: systolic blood pressure, DBP: diastolic blood pressure.

1997 to 2002. The study involved 4964 patients aged 7089 years with SBP ranging from 160 to $179 \mathrm{~mm} \mathrm{Hg}$ and/or DBP ranging from 90 to $99 \mathrm{~mm} \mathrm{Hg}$. Patients were assigned randomly to receive the angiotensin II receptor blocker (ARB) candesartan or a placebo, with open-label active antihypertensive therapy added as ( $84 \%$ of patients in the control group). The mean difference in blood pressure between the treatment group and control group was $3.2 \mathrm{~mm} \mathrm{Hg} \mathrm{SBP}$ and $1.6 \mathrm{~mm} \mathrm{Hg} \mathrm{DBP}$; the rates of dementia for the active treatment group and the control group were 6.3 and 6.8 cases per 1.000 patient-years, respectively, which is insignificant. In a subgroup analysis of SCOPE performed later, a significant 
positive effect on some cognitive domains (attention and episodic memory) was reported when using testing methods more sensitive than the MMSE.

3.5. HYVET-COG. The Hypertension in the very elderly trial-cognitive function assessment (HYVET-COG) examined antihypertensive medication for patients $\geq 80$ years of age. Eligible patients had no dementia, their SBP at entry was 160 to $200 \mathrm{~mm} \mathrm{Hg}$, and their DBP was below $110 \mathrm{~mm} \mathrm{Hg}$. Participants were randomly assigned to receive $1.5 \mathrm{mg}$ slow release diuretic (indapamide) with the option of ACEI (2$4 \mathrm{mg} /$ day, perindopril), or a placebo. The target SBP was $150 / 80 \mathrm{~mm} \mathrm{Hg}$. Possible cases of dementia (a fall in the MMSE score to $<24$ or a drop of three points in one year) were assessed by standard diagnostic criteria and expert review. HYVET-COG was the first randomized control study to report the effects of antihypertensive treatment in participants aged 80 years of age or older, finding a significant decrease in stroke after an average followup of 2.2 years, which led to its early termination. This followup period may be too short to detect any benefit preventing dementia. The mean difference in BP between the treatment and control groups was $15 \mathrm{~mm} \mathrm{Hg} \mathrm{SBP}$ and $5.9 \mathrm{~mm} \mathrm{Hg} \mathrm{DBP}$; the rates of dementia for active treatment group and the control group were 33 and 38 cases per 1.000 patient-years (hazard ratio 0.86; 95\% CI: 0.67-1.09), respectively, which is insignificant.

Of these five studies, only Syst-Eur and PROGRESS showed significant differences in the rate of dementia between the treatment and control groups.

However, when four of these data (Syst-Eur, PROGRESS, SHEP, and HYVET-COG) were combined in a meta-analysis [22], antihypertensive therapy was found to significantly reduce the risk of dementia (HR 0.87, 95\% CI: 0.76-1.00, $P=0.045)$. Another study, however, found that combining the antihypertensive therapy results from Syst-Eur, SHEP, and SCOPE reduced the risk of dementia by $11 \%$ (odds ratio 0.89 ; 95\% CI: 0.69-1.16), an insignificant effect [23]. Therefore, further long-term randomized trials, designed especially to assess a link between antihypertensive therapy and cognition as the primary outcome, are needed.

\section{Renin-Angiotensin-Aldosterone System and Cognitive Function}

Blocking the renin-angiotensin-aldosterone system (RAS) is another means that could have benefits on the prevention of dementia [24], but in a manner independent of BP lowering effect [25]. A potential neuroprotective effect on focal cerebral ischemia has been reported by blocking the RAS with an ARB that specifically targets the angiotensin II receptor [26]. Moreover, the Fournier hypothesis proposes that ARB treatment has potential advantages over ACEI treatment in the prevention of stroke and cognitive impairment because of the lower likelihood of harmful effects like vasoconstriction and proatherothrombogenesis while at the same time promoting neutral or even potentially beneficial effects like vasodilatation and endothelial modulation [27, $28]$.
Consistent with this theory, the ongoing telmisartan alone and in combination with ramipril global endpoint trial (ONTARGET) [29] and the parallel telmisartan randomized assessment study in ACEI intolerant subjects with cardiovascular disease (TRANSCEND) trial [30] have reported the effects of telmisartan, a unique ARB with peroxisome proliferator-activated receptor-gamma (PPARgamma-) stimulating activity, and the ACEI ramipril on cognitive function in patients aged 55 years and older with established atherosclerotic cardiovascular disease or diabetes with end-organ damage. In ONTARGET, a 56 month median duration month followup found cognitive impairment occurred in 652 (8\%) of the 7865 patients allocated ramipril, $584(7 \%)$ of the 7797 allocated telmisartan, and $618(8 \%)$ of the 7807 allocated a combination of the two (combination versus ramipril, odds ratio [OR] 0.95, 95\% CI $0.85-1.07, P=0.39$; telmisartan versus ramipril, OR 0.90, $0.80-1.01, P=0.06)$. Corresponding figures for cognitive decline were $1314(17 \%), 1279$ (17\%), and 1240 (17\%), respectively (telmisartan versus ramipril, OR 0.97, 0.89-1.06, $P=0.53$; combination versus ramipril, OR 0.95, 0.88-1.04, $P=0.28)$. In TRANSCEND, cognitive impairment occurred in $239(9 \%)$ of the 2694 participants allocated telmisartan compared with 245 (9\%) of the 2689 allocated a placebo (OR $0.97,0.81-1.17, P=0.76)$. The corresponding figures for cognitive decline were 454 (17\%) and 412 (16\%; OR 1.10, $0.95-1.27, P=0.22)$, respectively.

Recently, the prevention regimen for effectively avoiding second strokes (PRoFESS) trial [31] investigated the impact of ARBs on cognitive function in a randomized controlled design. There were no significant differences in the rate of cognitive decline or dementia between the treatment and control groups. These results were very similar to those in SCOPE. However, PRoFESS several limitations that obfuscate its conclusions. For example, the duration of the follow-up period was short, and there was a frequent discontinuation of the study drug among subjects, and many patients experienced recurrent stoke requiring termination of the antihypertensive treatments.

Figaro et al. [32] reported that antihypertensive therapy with an ARB and diuretic (telmisartan and hydrochlorothiazide) caused significant improvement in cognitive function compared to therapy using an ACEI and diuretic (lisinopril and hydrochlorothiazide). In addition, the observational study on cognitive function and systolic blood pressure reduction (OSCAR), an open label trial in 28 countries designed to evaluate the impact of the ARB eprosartan on cognitive function, found that a reduction in systolic blood pressure had an independent negative association with cognitive decline (odds ratio 0.77; 95\% CI: 0.73-0.82).

More recently, prospective cohort study of old (over 65) subjects demonstrated significantly lower hazard rates for incident dementia with ARBs than with an ACEI (hazard rate $0.81,95 \%$ CI $0.73-0.90)$ and other cardiovascular drugs $(0.76,0.69-0.84)$. However, the results may not be generalisable to women because women comprised only $2 \%$ of this cohort [33].

Although it is still not exactly clear how the ARBs confer this benefit, Tsukuda et al. [34] demonstrated that a low dose 
of telmisartan had a preventive effect on cognitive decline in an $\mathrm{AD}$ mouse model ( $\mathrm{A} \beta$-injection mouse model). This was in part due to the clearance of $A \beta$ in response to an inhibition of inflammation because of PPAR-gamma activation. Thus, ARBs that can act as a partial agonist for PPAR-gamma may provide a benefit for the treatment of dementia, along with their already blood pressure-lowering effects.

\section{Conclusion}

It is thought that there is a dependent relationship between the occurrence of HT and the risk of developing dementia in old age. This offers promise in the prevention of dementia because HT is a potentially reversible risk factor.

Recent epidemiological evidence suggests that some antihypertensive medications may reduce the risk for $\mathrm{AD}$. In particular, given that Syst-Eur found treating HT with the dihydropyridine CCB nitrendipine reduced the incidence of $\mathrm{AD}$ by $55 \%$, nitrendipine could prove to be a potentially reliable option for protection against dementia. Despite this encouraging evidence, several randomized trials have failed to support the efficacy of antihypertensive agents in $\mathrm{AD}$ dementia $[7,8]$. Thus, at present there is inconsistent evidence regarding the influence of antihypertensive drugs on dementia incidence and/or pathogenesis.

Because current theories assume $\mathrm{AD}$ is triggered by the accumulation of soluble and insoluble forms of $\beta$-amyloid, if high $\mathrm{BP}$ can increase the risk of $\mathrm{AD}$, they should then also lead to an accumulation of $\beta$-amyloid. In vitro study, using primary cortico-hippocampal neuron cultures generated from $\mathrm{AD}$ mouse model, at least 7 candidate antihypertensive agents including a calcium blocker, a $\beta$-adrenergic blocker, an $\alpha-\beta$ adrenergic blocker, a diuretic, a vasodilator and ARBs, that significantly reduced $\mathrm{AD}$-type $\beta$-amyloid protein $(\mathrm{A} \beta)$ accumulation [35]. It is thought that some of these drugs may have clinical benefits in protecting against progressive $\mathrm{A} \beta$-related memory disturbance in $\mathrm{AD}$.

Therefore, further prospective randomized studies comparing different antihypertensive classes are needed to provide more evidence regarding the effects of antihypertensive drugs on dementia risk and to determine whether certain antihypertensive classes provide greater benefits than others. In particular, whether these agents possess specific neuroprotective properties or increase cerebral perfusion remains to be clarified.

\section{Conflict of Interests}

The authors declare that there is no conflict of interests.

\section{References}

[1] C. Lenfant, A. V. Chobanian, D. W. Jones, and E. J. Roccella, "Seventh report of the Joint National Committee on the prevention, detection, evaluation, and treatment of high blood pressure (JNC 7): resetting the hypertension sails," Hypertension, vol. 41, no. 6, pp. 1178-1179, 2003.

[2] R. S. Vasan, A. Beiser, S. Seshadri et al., "Residual lifetime risk for developing hypertension in middle-aged women and men: the Framingham Heart Study," Journal of the American Medical Association, vol. 287, no. 8, pp. 1003-1010, 2002.

[3] W. B. Kannel, "Historic perspectives on the relative contributions of diastolic and systolic blood pressure elevation to cardiovascular risk profile," American Heart Journal, vol. 138, no. 3, pp. S205-S210, 1999.

[4] S. P. Kennelly, B. A. Lawlor, and R. A. Kenny, "Blood pressure and the risk for dementia-A double edged sword," Ageing Research Reviews, vol. 8, no. 2, pp. 61-70, 2009.

[5] F. Forette, M. L. Seux, J. A. Staessen et al., "The prevention of dementia with antihypertensive treatment: new evidence from the systolic hypertension in Europe (syst-eur) study," Archives of Internal Medicine, vol. 162, no. 18, pp. 2046-2052, 2002.

[6] C. Tzourio, C. Anderson, N. Chapman et al., "Effects of blood pressure lowering with perindopril and indapamide therapy on dementia and cognitive decline in patients with cerebrovascular disease," Archives of Internal Medicine, vol. 163, no. 9, pp. 1069-1075, 2003.

[7] W. B. Applegate, S. Pressel, J. Wittes et al., "Impact of the treatment of isolated systolic hypertension on behavioral variables: results from the systolic hypertension in the elderly program," Archives of Internal Medicine, vol. 154, no. 19, pp. 2154-2160, 1994.

[8] H. Lithell, L. Hansson, I. Skoog et al., "The study on cognition and prognosis in the elderly (SCOPE): principal results of a randomized double-blind intervention trial," Journal of Hypertension, vol. 21, no. 5, pp. 875-886, 2003.

[9] R. Peters, N. Beckett, F. Forette et al., "Incident dementia and blood pressure lowering in the Hypertension in the Very Elderly Trial cognitive function assessment (HYVET-COG): a double-blind, placebo controlled trial," The Lancet Neurology, vol. 7, no. 8, pp. 683-689, 2008.

[10] J. H. Chen, K. P. Lin, and Y. C. Chen, "Risk factors for dementia," Journal of the Formosan Medical Association, vol. 108, no. 10, pp. 754-764, 2009.

[11] P. B. Gorelick, "Risk factors for vascular dementia and Alzheimer disease," Stroke, vol. 35, no. 11, pp. 2620-2622, 2004.

[12] S. MacMahon, R. Peto, J. Cutler et al., "Blood pressure, stroke, and coronary heart disease-part 1: prolonged differences in blood pressure: prospective observational studies corrected for the regression dilution bias," Lancet, vol. 335, no. 8692, pp. 765-774, 1990.

[13] D. Knopman, L. L. Boland, T. Mosley et al., "Cardiovascular risk factors and cognitive decline in middle-aged adults," Neurology, vol. 56, no. 1, pp. 42-48, 2001.

[14] M. Kivipelto, E. L. Helkala, T. Hänninen et al., "Midlife vascular risk factors and late-life mild cognitive impairment: a population-based study," Neurology, vol. 56, no. 12, pp. 16831689, 2001.

[15] C. Reitz, M. X. Tang, J. Manly, R. Mayeux, and J. A. Luchsinger, "Hypertension and the risk of mild cognitive impairment," Archives of Neurology, vol. 64, no. 12, pp. 1734-1740, 2007.

[16] L. J. Launer, G. W. Ross, H. Petrovitch et al., "Midlife blood pressure and dementia: the Honolulu-Asia aging study," Neurobiology of Aging, vol. 21, no. 1, pp. 49-55, 2000.

[17] M. Yamada, Y. Mimori, F. Kasagi, T. Miyachi, T. Ohshita, and H. Sasaki, "Incidence and risks of dementia in Japanese women: radiation Effects Research Foundation Adult Health Study," Journal of the Neurological Sciences, vol. 283, no. 1-2, pp. 57-61, 2009.

[18] J. Birns and L. Kalra, "Cognitive function and hypertension," Journal of Human Hypertension, vol. 23, no. 2, pp. 86-96, 2009.

[19] C. Qiu, B. Winblad, and L. Fratiglioni, "The age-dependent 
relation of blood pressure to cognitive function and dementia," Lancet Neurology, vol. 4, no. 8, pp. 487-499, 2005.

[20] T. Ninomiya, T. Ohara, Y. Hirakawa et al., "Midlife and late-life blood pressure and dementia in japanese elderly: the hisayama study," Hypertension, vol. 58, no. 1, pp. 22-28, 2011.

[21] J. Li, Y. J. Wang, M. Zhang et al., "Chongqing Ageing Study GroupVascular risk factors promote conversion from mild cognitive impairment to Alzheimer disease," Neurology, vol. 76, no. 17, pp. 1485-1491, 2011.

[22] R. Peters, N. Beckett, F. Forette et al., "Incident dementia and blood pressure lowering in the Hypertension in the Very Elderly Trial cognitive function assessment (HYVET-COG): a double-blind, placebo controlled trial," The Lancet Neurology, vol. 7, no. 8, pp. 683-689, 2008.

[23] B. McGuinness, S. Todd, P. Passmore, and R. Bullock, "Blood pressure lowering in patients without prior cerebrovascular disease for prevention of cognitive impairment and dementia," Cochrane Database of Systematic Reviews, vol. 2, Article ID CD004034, 2009.

[24] C. Tzourio, C. Anderson, N. Chapman et al., "Effects of blood pressure lowering with perindopril and indapamide therapy on dementia and cognitive decline in patients with cerebrovascular disease," Archives of Internal Medicine, vol. 163, no. 9, pp. 1069-1075, 2003.

[25] F. Turnbull, B. Neal, M. Pfeffer et al., "Blood pressuredependent and independent effects of agents that inhibit the renin-angiotensin system," Journal of Hypertension, vol. 25, no. 5, pp. 951-958, 2007.

[26] M. Krikov, C. Thone-Reineke, S. Müller, A. Villringer, and T. Unger, "Candesartan but not ramipril pretreatment improves outcome after stroke and stimulates neurotrophin BNDF/TrkB system in rats," Journal of Hypertension, vol. 26, no. 3, pp. 544$552,2008$.

[27] A. Fournier, F. H. Messerli, J. M. Achard, and L. Fernandez, "Cerebroprotection mediated by angiotensin II: a hypothesis supported by recent randomized clinical trials," Journal of the American College of Cardiology, vol. 43, no. 8, pp. 1343-1347, 2004.

[28] F. Boutitie, R. Oprisiu, J. M. Achard et al., "Does a change in angiotensin II formation caused by antihypertensive drugs affect the risk of stroke? A meta-analysis of trials according to treatment with potentially different effects on angiotensin II," Journal of Hypertension, vol. 25, no. 8, pp. 1543-1553, 2007.

[29] The ONTARGET Investigators, "Telmisartan, ramipril, or both in patients at high risk for vascular events," The New England Journal of Medicine, vol. 358, no. 15, pp. 1547-1559, 2008.

[30] The Telmisartan Randomised AssessmeNt Study in ACE iNtolerant subjects with cardiovascular Disease (TRANSCEND) Investigators, "Effects of the angiotensin-receptor blocker telmisartan on cardiovascular events in highrisk patients intolerant to angiotensin-converting enzyme inhibitors: a randomised controlled trial," The Lancet, vol. 372, no. 9644, pp. 1174-1183, 2008.

[31] H. C. Diener, R. L. Sacco, S. Yusuf et al., "Effects of aspirin plus extended-release dipyridamole versus clopidogrel and telmisartan on disability and cognitive function after recurrent stroke in patients with ischaemic stroke in the Prevention Regimen for Effectively Avoiding Second Strokes (PRoFESS) trial: a double-blind, active and placebo-controlled study," The Lancet Neurology, vol. 7, no. 10, pp. 875-884, 2008.

[32] R. Fogari, A. Mugellini, A. Zoppi et al., "Effect of telmisartan/hydrochlorothiazide vs lisinopril/hydrochlorothiazide combination on ambulatory blood pressure and cognitive function in elderly hypertensive patients," Journal of Human Hypertension, vol. 20, no. 3, pp. 177-185, 2006.

[33] N. C. Li, A. Lee, R. A. Whitmer et al., "Use of angiotensin receptor blockers and risk of dementia in a predominantly male population: prospective cohort analysis," British Medical Journal, vol. 340, p. b5465, 2010.

[34] K. Tsukuda, M. Mogi, J. Iwanami et al., "Cognitive deficit in amyloid- $\beta$-injected mice was improved by pretreatment with a low dose of telmisartan partly because of peroxisome proliferator-activated receptor- $\gamma$ activation," Hypertension, vol. 54, no. 4, pp. 782-787, 2009.

[35] J. Wang, L. Ho, L. Chen et al., "Valsartan lowers brain $\beta$-amyloid protein levels and improves spatial learning in a mouse model of Alzheimer disease," Journal of Clinical Investigation, vol. 117, no. 11, pp. 3393-3402, 2007. 


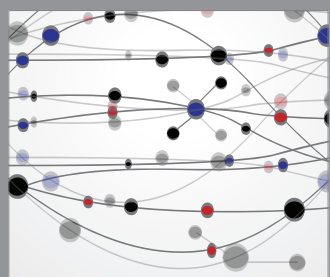

The Scientific World Journal
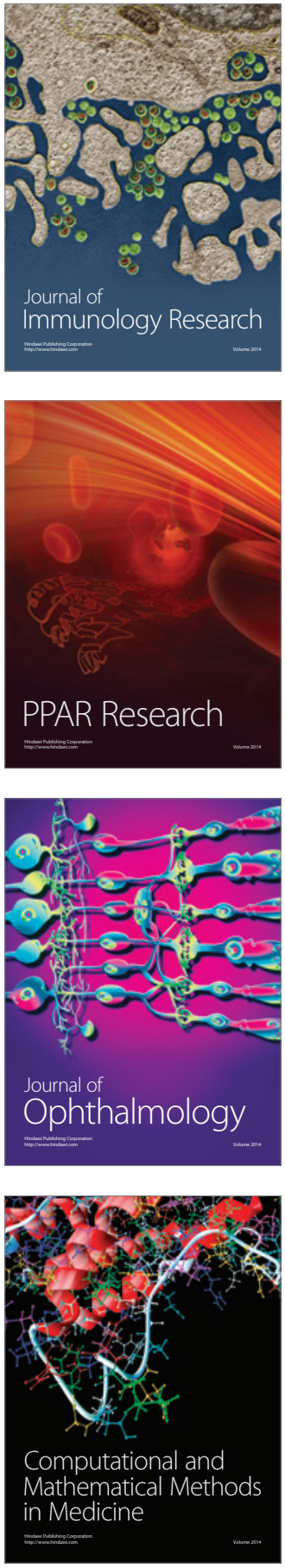

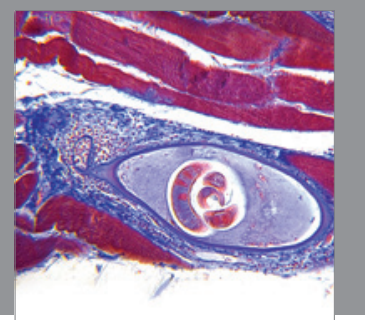

Gastroenterology

Research and Practice
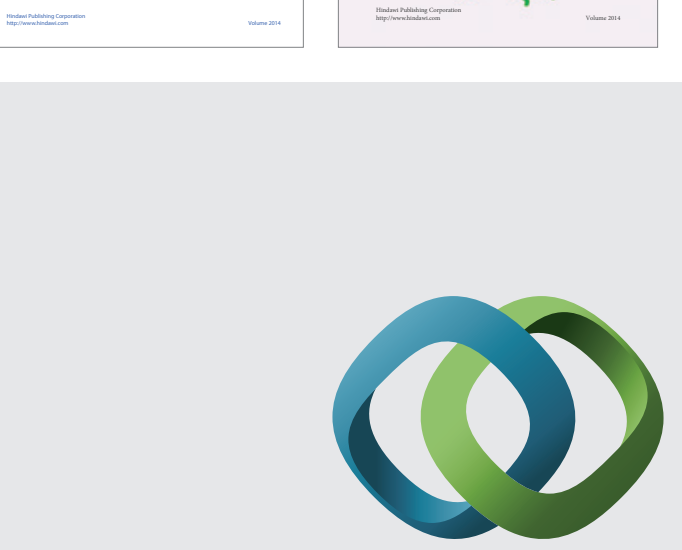

\section{Hindawi}

Submit your manuscripts at

http://www.hindawi.com
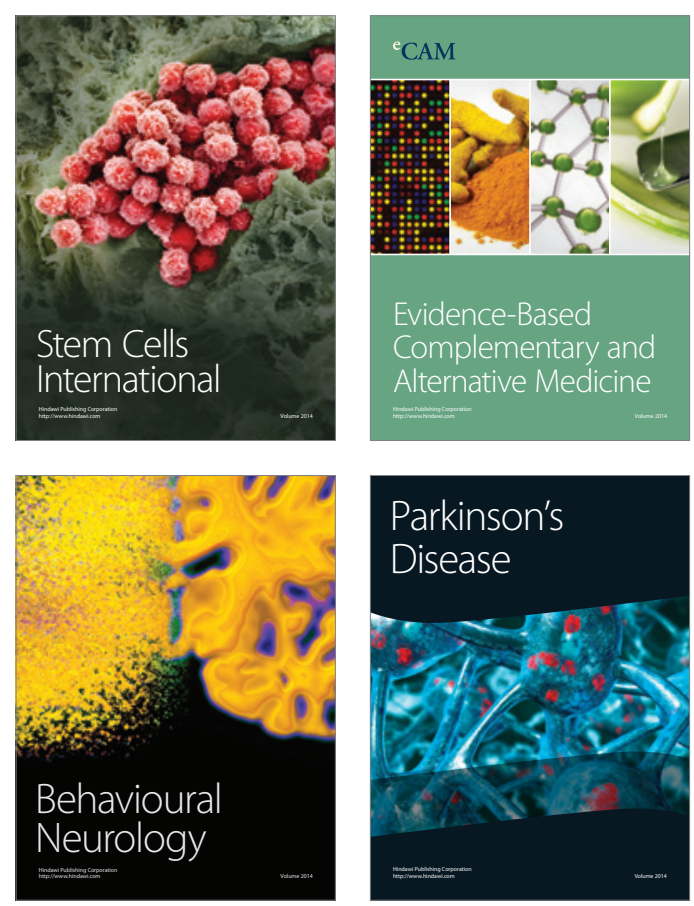

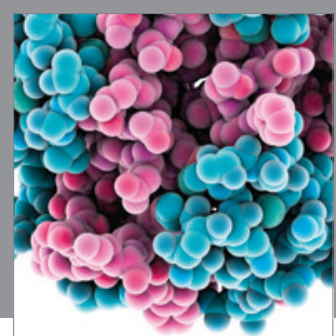

Journal of
Diabetes Research

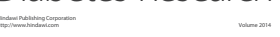

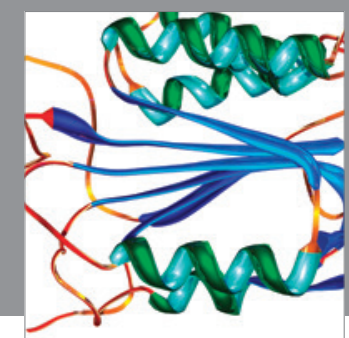

Disease Markers
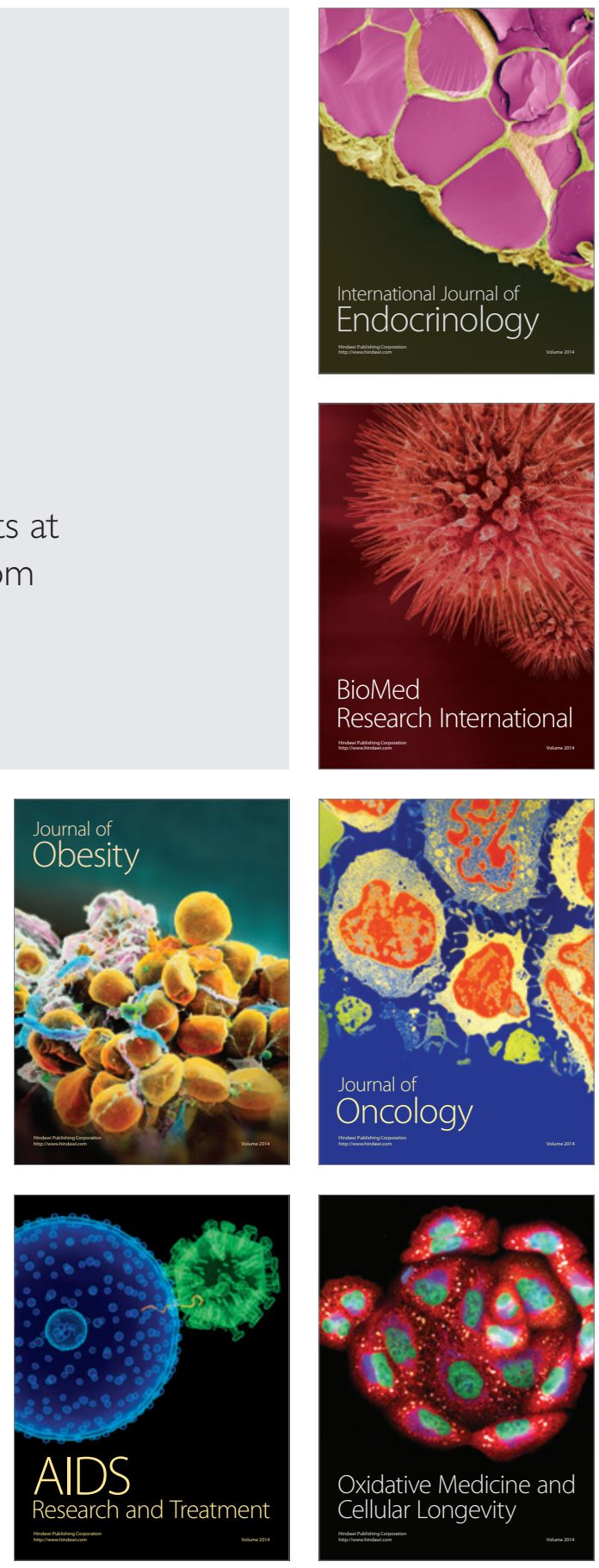